\title{
STOCHASTIC MONOTONICITY AND CONTINUITY PROPERTIES OF THE EXTINCTION TIME OF BELLMAN-HARRIS BRANCHING PROCESSES: AN APPLICATION TO EPIDEMIC MODELLING
}

\author{
M. GONZÁLEZ* AND \\ R. MARTÍNEZ, ${ }^{* *}$ University of Extremadura \\ M. SLAVTCHOVA-BOJKOVA, ${ }^{* * *}$ Bulgarian Academy of Sciences
}

\begin{abstract}
The aim of this paper is to study the stochastic monotonicity and continuity properties of the extinction time of Bellman-Harris branching processes depending on their reproduction laws. Moreover, we show their applications in an epidemiological context, obtaining an optimal criterion to establish the proportion of susceptible individuals in a given population that must be vaccinated in order to eliminate an infectious disease. First the spread of infection is modelled by a Bellman-Harris branching process. Finally, we provide a simulation-based method to determine the optimal vaccination policies.
\end{abstract}

Keywords: Bellman-Harris branching process; extinction time; vaccination policies; SIR; Monte Carlo method

2000 Mathematics Subject Classification: Primary 60J80

Secondary 92D30

\section{Introduction}

The Bellman-Harris branching process (BHBP) is a continuous-time model, which has been widely studied in the stochastic processes theory (see, for example, Chapter 4 of [3] for details). Moreover, from a practical outlook, it has been used to describe the evolution of populations over time in different situations, including, for example, to solve many problems related to cell populations (see, for example, [4], [5], [12], [17], [19], [26], and [27]).

It is well known that a BHBP becomes extinct or explodes to infinity depending on the mean value of its reproduction law. This property is inherited from its embedding Galton-Watson process (EGWP), leading us to the classification of subcritical, critical, and supercritical cases. Then, the extinction happens almost surely (a.s.) in the subcritical and critical cases, and has a positive probability in the supercritical case (obviously under the corresponding conditions to avoid trivial cases). However, the time necessary for the extinction of a BHBP cannot be deduced from its EGWP. This time is a random variable (RV) which depends on the continuous-time structure of the BHBP on its own. Even though the study of the extinction time is very interesting from both theoretical and practical view points, it has not been considered deeply enough (see, for example, [1], [10], [14], and [23]). In this paper we deal with this problem, investigating the dependence of the extinction time of a BHBP on its reproduction law. Moreover, we apply

Received 17 April 2008; revision received 12 November 2009.

* Postal address: Department of Mathematics, University of Extremadura, Badajoz 06006, Spain.

** Email address: rmartinez@unex.es

*** Postal address: Department of Probability and Statistics, Institute of Mathematics and Informatics, Bulgarian Academy of Sciences, Sofia 1113, Bulgaria. 
the obtained results in an epidemiological context. Actually, the problem of how to model the evolution of an infectious disease is very important and widely considered in the recent literature (see, for example, [8], [11], [15], [21], and [23]). However, in only a few papers (see, for example, [2], [7], [10], and [22]) has the waiting time to the extinction of the disease been used as a main tool to determine a vaccination policy. This is mainly because there are not enough results on this RV. In this work we propose a new approach to this topic.

The paper is organized as follows. In Section 2 we study some properties of the distribution function of the extinction time of a BHBP, mainly those related to stochastic monotonicity and continuity depending on its reproduction law. Then, in Section 3 we apply this study to investigate the behaviour of the extinction time of an infectious disease, depending on the proportion of immune individuals in the population. We consider diseases which follow an SIR (susceptible-infected-removed) scheme. It is well known that branching processes adequately fit this scheme (see [2] and [6]). So, first, we model the spread of infection by a BHBP. Then we study its extinction time distribution and propose an optimal vaccination level to immunize individuals in the population, based on the quantiles of such distributions. To guarantee the applicability of these results, we propose a simulation-based method which allows us to calculate the optimal proportion of susceptible individuals to be vaccinated. We also provide an illustrative example. Finally, to ease the reading, the proofs are presented in Section 4 .

\section{Properties of the extinction time}

In this section we study some properties related to the extinction time of BHBPs. First we obtain results for a BHBP with fixed reproduction law, which is referenced in terms of its probability generating function (PGF). Then, we study the properties of the extinction time of BHBPs with different reproduction laws, but with the same distribution of life length. Specifically, we establish stochastic monotonicity and continuity properties depending on the reproduction law.

To this aim, we denote by $T_{f}$ the extinction time of a BHBP, $\left\{Z_{t}\right\}_{t \geq 0}$, initiated at time 0 by a single individual, with reproduction law given by its PGF $f(\cdot)$ and life length with distribution function (DF) $G(\cdot)$ such that $G\left(0^{+}\right)=0$, i.e. there is a null probability of instantaneous death. Mathematically, we have

$$
T_{f}=\inf \left\{t \geq 0: Z_{t}=0\right\}
$$

where $Z_{t}$ denotes the number of individuals in the population at time $t$. Intuitively, $T_{f}$ is the maximal time that the population survives when the PGF of the reproduction law is $f(\cdot)$.

Fix the PGF $f(\cdot)$. We denote by $v_{f}(\cdot)$ the DF of the extinction time $T_{f}$, i.e.

$$
v_{f}(t)=\mathrm{P}\left(T_{f} \leq t\right), \quad t \in \mathbb{R} .
$$

Since $G\left(0^{+}\right)=0$, then $v_{f}(0)=0$. Furthermore, using the methods given in [3, Theorem IV.2.1, p. 139], it is easy to deduce that $v_{f}(\cdot)$ is the unique bounded function that satisfies the integral equation

$$
v_{f}(t)= \begin{cases}0, & t<0, \\ \int_{0}^{t} f\left(v_{f}(t-s)\right) \mathrm{d} G(s), & t \geq 0 .\end{cases}
$$

Moreover, let $q_{f}$ be the extinction probability of a BHBP started with one ancestor and with reproduction law given by the PGF $f(\cdot)$. It is clear that $q_{f}=\mathrm{P}\left(T_{f}<\infty\right)$, and it is also well 
known that $q_{f}=1$ if and only if $m_{f} \leq 1$, where $m_{f}$ denotes the reproduction mean associated to $f(\cdot)$. So that, for such a PGF $f(\cdot)$ with $m_{f}>1, v_{f}(\cdot)$ is the DF of a nonproper RV because $\mathrm{P}\left(T_{f}<\infty\right)<1$. In any case, it follows that

$$
\tilde{v}_{f}(t)=\mathrm{P}\left(T_{f} \leq t \mid T_{f}<\infty\right)=\frac{v_{f}(t)}{q_{f}}, \quad t \geq 0,
$$

and, from (2.1), it is easy to show that $\widetilde{v}_{f}(\cdot)$ also satisfies the equation

$$
\widetilde{v}_{f}(t)=\int_{0}^{t} g\left(\widetilde{v}_{f}(t-s)\right) \mathrm{d} G(s), \quad t \geq 0,
$$

where $g(s)=q_{f}^{-1} f\left(q_{f} s\right)$ is a PGF such that $m_{g}<1$, that is, $\widetilde{v}_{f}(t)=v_{g}(t)$ for all $t \in \mathbb{R}$. Therefore, without loss of generality, from now on, in many situations we can consider a PGF $f(\cdot)$ such that the extinction time $T_{f}$ is a proper RV, i.e. $m_{f} \leq 1$.

The DF $v_{f}(\cdot)$ inherits some properties of the DF $G(\cdot)$ as follows. Both of them have support on the nonnegative real numbers. Moreover, if the DF of the life length $G(\cdot)$ is discrete, then the DF of the extinction time $v_{f}(\cdot)$ is also discrete. For the absolutely continuous case, we obtain the analogous result.

Proposition 2.1. If $G(\cdot)$ is an absolutely continuous DF then $v_{f}(\cdot)$ is also an absolutely continuous DF.

The DF $v_{f}(\cdot)$ is determined implicitly from (2.1). However, it is useful to obtain procedures which allow us to know or at least to approximate the value of this function at each point $t$. To this end, we introduce the functional operator $H_{f}(\cdot)$, defined on any function $u(\cdot)$ from the nonnegative real numbers $\mathbb{R}_{+}$to the closed interval $[0,1]$ :

$$
H_{f}(u)(t)=\int_{0}^{t} f(u(t-s)) \mathrm{d} G(s), \quad t \geq 0 .
$$

Also, for all $n \geq 1$, we denote by $H_{f}^{n}(\cdot)$ the $n$th composition of the operator $H_{f}(\cdot)$, that is, $H_{f}^{n+1}(u)(\cdot)=H_{f}\left(H_{f}^{n}(u)\right)(\cdot), n=1,2, \ldots$, and $H_{f}^{1}(u)(\cdot)=H_{f}(u)(\cdot)$. Using this notation, from $(2.1)$ we find that $v_{f}(\cdot)$ is the unique bounded function satisfying the fixed-point equation $u(\cdot)=H_{f}(u)(\cdot)$. We also derive the following result.

Theorem 2.1. If $f(\cdot)$ is a PGF then, for each function $h: \mathbb{R}_{+} \rightarrow[0,1]$, it is verified that

$$
v_{f}(t)=\lim _{n \rightarrow \infty} H_{f}^{n}(h)(t), \quad t \geq 0 .
$$

This result, besides giving us a way to approximate the DF $v_{f}(\cdot)$ at each point, provides a useful tool to investigate the behaviour of the extinction times for BHBPs with different reproduction laws and the same life-length distribution. So, next we consider the behaviour of $v_{f}(\cdot)$ depending on $f(\cdot)$, when $G(\cdot)$ is fixed.

Theorem 2.2. Let $f(\cdot)$ and $g(\cdot)$ be PGFs. If $f(s) \leq g(s)$ for all $0 \leq s \leq 1$ then $v_{f}(t) \leq v_{g}(t)$ for all $t \geq 0$.

Remark 2.1. It is not hard to show that if the reproduction law given by $f(\cdot)$ is stochastically greater than that given by $g(\cdot)$ then $f(s) \leq g(s)$ for all $0 \leq s \leq 1$. But, in general, the vice versa statement is not true. 
From the previous theorem we deduce that the condition $f(s) \leq g(s)$ for all $0 \leq s \leq 1$ implies that the extinction time of the BHBP with PGF $f(\cdot)$ is stochastically greater than that of the BHBP with PGF $g(\cdot)$, i.e. the monotonicity property of the PGFs is inherited by the DF of the extinction time.

Now we show in the following result that minor changes in the PGF $f(\cdot)$ generates minor changes in the extinction time.

Theorem 2.3. Let $f(\cdot)$ be a PGF such that $m_{f}<1$. For each $\varepsilon>0$, there exists $\delta=$ $\delta(\varepsilon, f)>0$ such that if $g(\cdot)$ is a PGF satisfying

$$
\sup _{0 \leq s \leq 1}|f(s)-g(s)| \leq \delta
$$

then

$$
\sup _{0 \leq t<\infty}\left|v_{f}(t)-v_{g}(t)\right| \leq \varepsilon .
$$

Remark 2.2. (i) It is important to point out that, given a PGF, it is possible to find another arbitrarily close PGF. Actually, for fixed $f(\cdot)$ and any $\delta>0$, there exists a PGF $g(\cdot)$ such that $\sup _{0 \leq s \leq 1}|f(s)-g(s)| \leq \delta$. Indeed, since $f(\cdot)$ is a uniformly continuous function on the interval [0,1], then there exists $0<\alpha<1$ such that $\left|f(s)-f\left(s^{*}\right)\right| \leq \delta$ for all $s, s^{*}$ with $0 \leq s, s^{*} \leq 1$ and $\left|s-s^{*}\right| \leq \alpha$. For each $0 \leq s \leq 1$, let $g(s)=f(\alpha+(1-\alpha) s)$. We will show in the next section that $g(\cdot)$ is a PGF. Since $\alpha+(1-\alpha) s-s \leq \alpha$ for all $0 \leq s \leq 1$, then $\sup _{0 \leq s \leq 1}|f(s)-g(s)| \leq \delta$.

(ii) In Theorem 2.3 specifically, we proved a continuity property for the DF $v_{f}(\cdot)$ depending on $f(\cdot)$, when $m_{f}<1$. Taking into account (2.2), we can also deduce this continuity property when $m_{f}>1$. Indeed, let $f(\cdot)$ be a PGF such that $m_{f}>1$. From the embedded generation process associated with the BHBP and the equation $f\left(q_{f}\right)=q_{f}$, it is not hard to obtain the continuity of $q_{f}$ depending on $f(\cdot)$. Moreover, since $v_{f}(t)=q_{f} v_{g}(t)$, recall that $g(s)=q_{f}^{-1} f\left(q_{f} s\right)$ is a PGF such that $m_{g}<1$, then, from Theorem 2.3 , the continuity property can be proved.

\section{Application to epidemic modelling}

Branching processes have been widely used to model the evolution of infectious diseases that follow SIR schemes, at least in their early stages (see, for example, [2], [6], [13], [18], [21], and [24]). In particular, infectious diseases with long incubation periods and negligible contagious times, such as avian flu, measles, and mumps, can be described by a BHBP.

To model the spread of an infectious disease using a BHBP, we consider the following scheme. Let us assume that three types of individual exist in the population: infected individuals, healthy individuals who are susceptible to catching the infection (susceptible individuals), and healthy individuals who are immune to the infection. The disease is spread when an infected individual is in contact with susceptible individuals. Note that, during the incubation period, an infected individual does not show any symptoms of the disease and does not pass the disease on to a susceptible individual. Moreover, when the infectious disease is observed in an individual, this individual is either isolated (for example, in human or animal populations) or culled (for example, in animal populations with very dangerous diseases), so that the individual ceases to be infective. Hence, just after the incubation period and before being isolated or culled, there is a very short contact period (in comparison with the incubation period) in which the individual may infect others. We denote by $p_{k}$ the probability that one infected individual contacts $k$ healthy individuals, $k \geq 0$, and we denote by $\alpha(0 \leq \alpha \leq 1)$ 
the proportion of immune individuals in the population. We suppose that both infected and immune individuals are dispersed uniformly in the population. Furthermore, we assume that the population size is fixed and large enough in comparison with the number of infected individuals, so that $\alpha$ and the contact distribution law, $\left\{p_{k}\right\}_{k \geq 0}$, can be considered stable over time (see [15]). Note that this is not a restriction in either critical and subcritical processes, because of their almost-sure extinction, or in the early stages of supercritical processes.

Under these assumptions, the probability that an infected individual transmits the disease to $k$ susceptible individuals, where $\alpha$ is the proportion of immune individuals in the population, is given by

$$
p_{\alpha, k}=\sum_{j=k}^{\infty}\left(\begin{array}{l}
j \\
k
\end{array}\right) \alpha^{j-k}(1-\alpha)^{k} p_{j},
$$

i.e. the infected individual has been in contact with $j$ healthy individuals and among them there have been $k$ susceptible individuals. We call $\left\{p_{\alpha, k}\right\}_{k \geq 0}$ the infection distribution law when the proportion of immune individuals in the population is $\alpha$. Note that if no individual in the population is immune, i.e. $\alpha=0$, then every individual will be infected whenever he/she contacts an infected individual, i.e. $p_{0, k}=p_{k}$ for all $k \geq 0$. On the other hand, if all individuals in the population are immune, i.e. $\alpha=1$, then the infection does not spread, i.e. $p_{1, k}=0$ for all $k>0$. Following this spreading scheme along time, infected individuals pass on the disease to other susceptible individuals, and so on. We model the number of infected individuals in a population with a proportion $\alpha$ of immune individuals by a BHBP, such that its offspring law is determined by the infection distribution law $\left\{p_{\alpha, k}\right\}_{k \geq 0}$ and the DF of the life length of an infected individual is given by an arbitrary DF $G(\cdot)$ of a nonnegative RV. By life length we mean the period (measured in real time) consisting of the incubation period plus the comparatively very short (negligible) contact period. Note that we assume that the life length of an infected individual does not depend on either the proportion of immune individuals in the population or the contact distribution law.

In order to immunize a proportion of susceptible individuals, we suppose that a vaccination policy is applied. Our objective is to determine what proportion, $\alpha$, of these individuals might be vaccinated/immunized to guarantee the extinction of the disease, possibly in a given period of time. We call this proportion the vaccination level. Specifically, we deal with the problem of determining the optimal vaccination level, which depends not only on the speed of the transmission of the disease, expressed in terms of the infection distribution law $\left\{p_{\alpha, k}\right\}_{k \geq 0}$, but also on the time until the epidemic becomes extinct after the vaccination process finishes. To this end, we first study the behaviour of the extinction time of the epidemic depending on the vaccination level, applying the results of the previous sections. Then, from this study we propose an optimal vaccination level, and, finally, we illustrate how to determine this optimal vaccination level by means of a simulation method.

\subsection{The extinction time of the epidemic}

In what follows, our goal is to investigate the distribution of the extinction time of a BHBP depending on the vaccination level $\alpha$. To this end, for each $\alpha$ such that $0 \leq \alpha \leq 1$, we denote by $f_{\alpha}(\cdot)$ the PGF of $\left\{p_{\alpha, k}\right\}_{k \geq 0}$. From (3.1), it is easy to obtain

$$
f_{\alpha}(s)=f(\alpha+(1-\alpha) s), \quad 0 \leq s \leq 1,
$$

where $f(\cdot)$ is the PGF of $\left\{p_{k}\right\}_{k \geq 0}$. Moreover, we denote by $T_{\alpha}$ the extinction time of a BHBP initiated at time 0 with a single infected individual and with PGF $f_{\alpha}(\cdot)$, and we denote by $v_{\alpha}(\cdot)$ 
the DF of $T_{\alpha}$. Intuitively, $T_{\alpha}$ is the maximal time that the infection survives in the population when the proportion of immune individuals is $\alpha$.

Also, we denote by $m$ the mean number of contacts of an infected individual and by $m_{\alpha}$ the mean number of susceptible individuals, who are infected by a contagious individual, given that the proportion of immune individuals in the population is $\alpha$. Then, from (3.1), it is easy to calculate that

$$
m_{\alpha}=(1-\alpha) m
$$

Taking into account (3.3), $m_{\alpha} \leq 1$ is equivalent to $\max \left\{0,1-m^{-1}\right\} \leq \alpha \leq 1$, which depends on the mean number of contacts of an infected individual. In order to simplify the notation, from now on we denote by $\alpha_{\text {inf }}=\max \left\{0,1-m^{-1}\right\}$ the smallest proportion of immune individuals, so that the infectious disease becomes extinct a.s.

From the properties of $f(\cdot),(3.2)$, and Theorems 2.2 and 2.3, it is not hard to show that, for each $t \geq 0$, the function $v_{\alpha}(t)$ is nondecreasing and continuous on $\alpha$ for $\alpha_{\text {inf }}<\alpha \leq 1$, i.e. in a continuous way, the greater the proportion of immune individuals, the more probable it is that the infectious disease disappears faster.

Furthermore, some parameters of $T_{\alpha}$ inherit these properties of $v_{\alpha}(\cdot)$. Next we investigate the monotonicity and continuity properties of the quantiles of the distribution of the infected extinction time, depending on the proportion of immune individuals in the population.

For fixed $\alpha$ and $p$, with $\alpha_{\text {inf }} \leq \alpha \leq 1$ and $0<p<1$, we denote by $t_{p}^{\alpha}$ the quantile of order $p$ of the variable $T_{\alpha}$. We have the following result.

Theorem 3.1. Let $p$ be such that $0<p<1$.

(i) If $\alpha_{\mathrm{inf}} \leq \alpha_{1}<\alpha_{2} \leq 1$ then $t_{p}^{\alpha_{2}} \leq t_{p}^{\alpha_{1}}$.

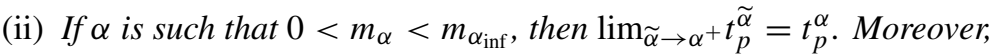

(a) if $v_{\alpha}\left(t_{p}^{\alpha}\right)=p$ then $t_{p}^{\alpha} \leq \lim _{\widetilde{\alpha} \rightarrow \alpha^{-}} t_{p}^{\widetilde{\alpha}} \leq t^{*}$, with $t^{*}=\sup \left\{t: v_{\alpha}(t)=p\right\}$;

(b) if $v_{\alpha}\left(t_{p}^{\alpha}\right)>p$ then $\lim _{\widetilde{\alpha} \rightarrow \alpha^{-}} t_{p}^{\widetilde{\alpha}}=t_{p}^{\alpha}$;

(c) if $v_{\alpha}(\cdot)$ is an increasing and absolutely continuous function then $\lim _{\widetilde{\alpha} \rightarrow \alpha} t_{p}^{\widetilde{\alpha}}=t_{p}^{\alpha}$.

Remark 3.1. Note that if $G(\cdot)$ is an increasing and absolutely continuous function defined on the nonnegative real numbers, we deduce from Proposition 2.1 that $v_{\alpha}(\cdot)$ is also of the same type and, therefore, for $\alpha_{\text {inf }}<\alpha \leq 1, t_{p}^{\alpha}$ is a continuous function depending on $\alpha$.

\subsection{Determining vaccination policies}

When an infectious disease is strongly detrimental to the population within which it is spreading, such that it becomes an epidemic, then a vaccination policy should be applied to protect the susceptible individuals and terminate the epidemic. Since, in most cases, it is impossible to immunize the whole population, only a proportion of susceptible individuals can be immunized through vaccination. How this proportion is determined is an important problem which depends on a number of factors. A significant factor for public authorities to assess the vaccination efficiency is the time that the infectious disease should be allowed to survive after vaccination.

In what follows we propose an optimal proportion of susceptible individuals to be immunized. Without loss of generality, we suppose that, before vaccination, every healthy individual in contact with an infected individual is not immune, i.e. the contact always produces the infection. Then, before the vaccination, with probability $p_{k}$, an infected individual passes the disease on 
to $k$ susceptible individuals. Moreover, after the vaccination process, we suppose that every vaccinated individual is immune to the infectious disease. If at the end of the vaccination process we have a proportion $\alpha$ of vaccinated susceptible individuals then, with probability $p_{\alpha, k}$ (see (3.1)), an infected individual transmits the disease to $k$ susceptible individuals.

To guarantee the extinction of the disease a.s., $\alpha$ should be equal to at least $\alpha_{\text {inf }}$. Intuitively, it is clear that increasing the vaccination level leads to a decrease (stochastically) in the extinction time of the infection. Obviously, the best strategy is to vaccinate all the population, but this is not practically feasible in most cases. This is why we propose a possible way of defining the optimal proportion of vaccinated individuals, to guarantee that the infection terminates by a given instant of time after the vaccination process ends. The vaccination policy is based on the quantiles of the extinction time $T_{\alpha}$. For fixed $p$ and $t$, with $0<p<1$ and $t>0$, we look for vaccination policies which guarantee that the infectious disease becomes extinct, with probability greater than or equal to $p$, not later than time $t$ after the vaccination process has ended. Let us suppose that we have vaccinated a proportion $\alpha$ of susceptible individuals. If at the end of the vaccination process there is a single infected individual in the population, since this infected individual might have already lived some time before, then the probability that the disease becomes extinct no later than time $t$ after the vaccination process has ended is greater than or equal to $v_{\alpha}(t)$. In Appendix A we provide a mathematical justification of this fact.

On the other hand, if there are $z$ infected individuals at the end of the vaccination process, since each individual reproduces/infects independently from the others, then the probability that the disease becomes extinct no later than time $t$ after the vaccination process has ended can be bounded by $\left(v_{\alpha}(t)\right)^{z}$.

Consequently, any vaccination level $\alpha$ such that $v_{\alpha}(t) \geq p^{(z)}$ or, equivalently, $t_{p^{(z)}}^{\alpha} \leq t$, with $p^{(z)}=p^{1 / z}$, could be used. Taking this fact into account, we propose as the optimal vaccination policy that one which corresponds to the smallest $\alpha$ of all of them, i.e.

$$
\begin{aligned}
\alpha_{q} & =\alpha_{q}(p, t, z) \\
& =\inf \left\{\alpha: \alpha_{\text {inf }} \leq \alpha \leq 1, v_{\alpha}(t) \geq p^{(z)}\right\} \\
& =\inf \left\{\alpha: \alpha_{\text {inf }} \leq \alpha \leq 1, t_{p^{(z)}}^{\alpha} \leq t\right\}
\end{aligned}
$$

Applying the monotonicity and continuity properties of the functions $v_{\alpha}(t)$ and $t_{p}^{\alpha}$ (depending on $\alpha$ ) we have $v_{\alpha_{q}}(t) \geq p^{(z)}$ and $t_{p^{(z)}}^{\alpha_{q}} \leq t$ if $\alpha_{q}>\alpha_{\text {inf }}$. Note that, since $\left(v_{\alpha}(t)\right)^{z}$ is a lower bound of the probability of interest, then some $\alpha$ less than $\alpha_{q}$ could also be valid for our general aim. Moreover, although $t$ and $p$ are fixed arbitrarily, in order to find a solution of the problem, it is necessary that $t \geq t_{p^{(z)}}^{1}$ or, equivalently, $p^{(z)} \leq v_{1}(t)$.

\subsection{A simulation-based method for determining vaccination policies}

In the previous subsections we proposed a vaccination policy defined by $\alpha_{q}$. This vaccination policy depends on the DF of the extinction time. Therefore, to calculate $\alpha_{q}$, it is necessary to know $v_{\alpha}(\cdot)$ for $\alpha$ such that $\alpha_{\text {inf }} \leq \alpha \leq 1$. Although $v_{\alpha}(\cdot)$ satisfies (2.1) and (2.3), in general it is not possible to obtain this function in a closed form. Recently, some numeric and simulation methods have been provided in order to approximate the function satisfying (2.1) (see, for example, [20]). In what follows we determine $\alpha_{q}$, approximating $v_{\alpha}(\cdot)$ by means of a simulation-based method when $\left\{p_{k}\right\}_{k \geq 0}$ and $G(\cdot)$ are known. When $\alpha$ is fixed, such that $\alpha_{\text {inf }} \leq \alpha \leq 1$, we apply the Monte Carlo method to estimate the empirical DF of the extinction time when the proportion of immune individuals is $\alpha$. Taking different $\alpha$ s sufficiently close, we approach $\alpha_{q}$ from its definition. To simulate the spread of the disease when the proportion 
of immune individuals is $\alpha$, it is enough to know $G(\cdot)$ and $\left\{p_{k}\right\}_{k \geq 0}$. Usually, the life-length distribution and the contact distribution law are estimated from the information that becomes available as the epidemic proceeds (see, for example, [16]).

Next we illustrate the simulation-based method by means of the following example. Let the life length of an infected individual follow the gamma distribution $\Gamma(2,1)$. Also, let the contact distribution law follow a Poisson distribution with parameter $m$. These types of distribution have been used to model the incubation period and the number of contacts for infectious diseases (see, for example, [10], [11], and [21]). From (3.2) we have

$$
f_{\alpha}(s)=f(\alpha+(1-\alpha) s)=\mathrm{e}^{-m(1-\alpha-(1-\alpha) s)}=\mathrm{e}^{-m_{\alpha}(1-s)}, \quad 0 \leq s \leq 1,
$$

which means that the infection distribution law also follows a Poisson distribution with parameter $m_{\alpha}=(1-\alpha) m$, where $m_{\alpha}$ is the number of susceptible individuals that become infected. Note that, for fixed $m, \alpha$ is determined one-to-one by $m_{\alpha}$. Therefore, instead of calculating $\alpha_{q}$, we determine $m_{q}=\left(1-\alpha_{q}\right) m$. From the definition of $\alpha_{q}$ we obtain

$$
m_{q}=m_{q}(t, p, z)=\sup \left\{m_{p}: 0 \leq m_{p} \leq 1, u_{m_{p}}(t) \geq p^{(z)}\right\},
$$

where $u_{m_{p}}(\cdot)$ is the DF of the extinction time when the infection distribution law follows a Poisson distribution with parameter $m_{p}$. Note that $v_{\alpha}(\cdot)=u_{m_{\alpha}}(\cdot)$ and that $m_{q}$ is independent of the magnitude of $m$. Therefore, to approximate $m_{q}$ we need to obtain only the empirical distribution $u_{m_{p}}(\cdot)$ for $0 \leq m_{p} \leq 1$ using the Monte Carlo method. To this end, for each fixed $m_{p}, 10000$ processes have been simulated and their extinction times have been calculated. The left-hand diagram of Figure 1 shows the behaviour of the empirical DF $u_{m_{p}}(\cdot)$ for several $m_{p}$ s. Note that, as $m_{p}$ increases, the extinction time also increases (stochastically).

As an example, to compute $m_{q}$, we take $p=0.9, t=15$, and $z=3$. Then we have $p^{(z)}=0.965$. The behaviour of the estimated value of $u_{m_{p}}(15)$, jointly with an upper confidence bound of $95 \%$, depending on $m_{p}$, is given in the right-hand diagram of Figure 1. We also show in Figure 1, using dashed lines, that, given $p^{(z)}=0.965$, an approximation of $m_{q}(15,0.9,3)$ is 0.64 .
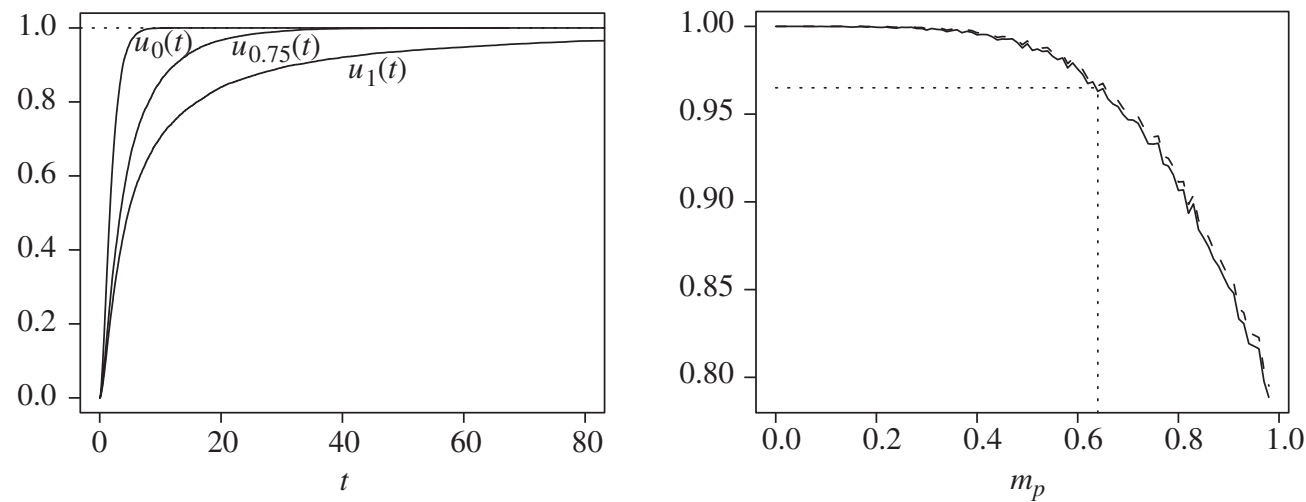

FiguRe 1: Left: behaviour of the empirical distribution functions of $u_{m_{p}}(\cdot)$ depending on $m_{p}$. Right: behaviour of the estimated value of $u_{m_{p}}(15)$, jointly with an upper confidence bound of $95 \%$, depending on $m_{p}$ (dashed line). 


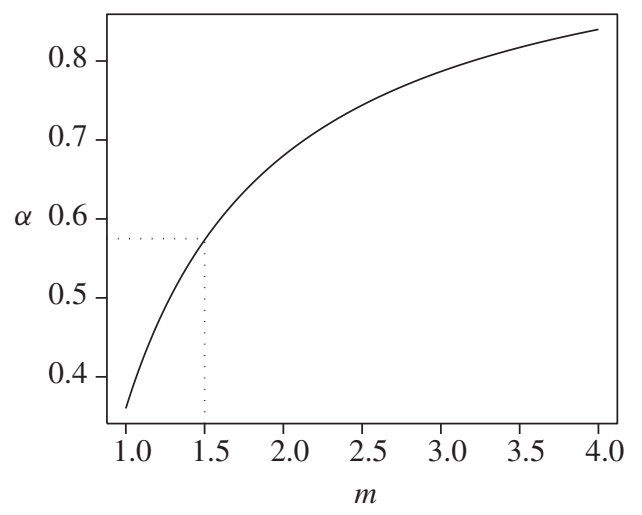

FIGURE 2: Proportion of individuals to be vaccinated, depending on $m$ and taking into account $m_{q}(15,0.9,3)=0.64$.

Finally, in Figure 2 we illustrate the proportion of individuals to be vaccinated, depending on $m$ and taking into account $m_{q}(15,0.9,3)$. Note that, if the mean number of contacts per individual, $m$, is close to 1.5 then we need to vaccinate about $57 \%$ of the population in order to guarantee that the infectious disease becomes extinct with probability greater than or equal to 0.9 not later than time 15 after the vaccination period has ended.

Remark 3.2. For the computer simulations, we used the language and environment of statistical computing and graphics, $\mathbf{R}$ (see [25]).

\section{Proofs}

In this section we provide the proofs of the results in the paper.

Proof of Proposition 2.1. For all $t \geq 0$, we have

$$
\begin{aligned}
v_{f}(t) & =\int_{0}^{t} f\left(v_{f}(t-s)\right) \mathrm{d} G(s) \\
& =f(0) G(t)+(1-f(0)) \int_{0}^{t} F_{f}(t-s) \mathrm{d} G(s),
\end{aligned}
$$

with $F_{f}(y)=(1-f(0))^{-1}\left(f\left(v_{f}(y)\right)-f(0)\right)$ for $y \geq 0$. Since $f(\cdot)$ is a PGF and $v_{f}(\cdot)$ is a $\mathrm{DF}$, then $F_{f}(\cdot)$ is also a DF on the nonnegative real numbers, and, therefore,

$$
\int_{0}^{t} F_{f}(t-s) \mathrm{d} G(s)=\int_{0}^{\infty} F_{f}(t-s) \mathrm{d} G(s)=\left(F_{f} * G\right)(t)
$$

is the convolution of $F_{f}(\cdot)$ and $G(\cdot)$. If $G(\cdot)$ is an absolutely continuous DF then it is well known that $F_{f} * G(\cdot)$ is also an absolutely continuous DF (see [9, p. 272]). Therefore, since $v_{f}(\cdot)$ is a convex linear combination of two absolutely continuous DFs, then it is also an absolutely continuous DF.

Proof of Theorem 2.1. Let $h(\cdot)$ be a function from $\mathbb{R}_{+}$to the closed interval $[0,1]$. In order to obtain the result, it is enough to prove the following four statements.

(S1) For all $t \geq 0, \widetilde{G}(t) \leq H_{f}(h)(t) \leq G(t)$, with $\widetilde{G}(t)=f(0) G(t)$. 
(S2) $H_{f}(\cdot)$ is a nondecreasing operator, i.e. if $h_{i}: \mathbb{R}_{+} \rightarrow[0,1], i \in\{1,2\}$, are two functions such that $h_{1}(t) \leq h_{2}(t)$ for all $t \geq 0$, then $H_{f}\left(h_{1}\right)(t) \leq H_{f}\left(h_{2}\right)(t)$ for all $t \geq 0$.

(S3) For all $t \geq 0$, there exist $u_{1}(t)=\lim _{n \rightarrow \infty} H_{f}^{n}(\widetilde{G})(t)$ and $u_{2}(t)=\lim _{n \rightarrow \infty} H_{f}^{n}(G)(t)$.

(S4) Let $u_{1}(\cdot)$ and $u_{2}(\cdot)$ be solutions of the fixed-point equation $u(\cdot)=H_{f}(u)(\cdot)$. Then $v_{f}(\cdot)=u_{1}(\cdot)=u_{2}(\cdot)$.

Indeed, from these statements, it is easy to prove that, for all $n \geq 1$ and $t \geq 0$,

$$
\begin{aligned}
v_{f}(t) & =u_{1}(t) \\
& =\lim _{n \rightarrow \infty} H_{f}^{n}(\widetilde{G})(t) \\
& \leq \lim _{n \rightarrow \infty} H_{f}^{n+1}(h)(t) \\
& \leq \lim _{n \rightarrow \infty} H_{f}^{n}(G)(t) \\
& =u_{2}(t)=v_{f}(t) .
\end{aligned}
$$

It remains to prove statements (S1)-(S4).

(S1) Since $f(\cdot)$ is an increasing function such that $f(1)=1$, we have

$$
\widetilde{G}(t)=f(0) G(t) \leq \int_{0}^{t} f(h(t-s)) \mathrm{d} G(s) \leq G(t) .
$$

(S2) Since $f(\cdot)$ is an increasing function and $h_{1}(t) \leq h_{2}(t)$ for all $t \geq 0$, then the statement is shown.

(S3) By (S1), (S2), and taking iterations, for each $t \geq 0,\left\{H_{f}^{n}(\widetilde{G})(t)\right\}_{n \geq 1}$ is an upper bounded, nondecreasing sequence and $\left\{H_{f}^{n}(G)(t)\right\}_{n \geq 1}$ is a lower bounded, nonincreasing sequence. Hence, the statement follows.

(S4) Since $f(\cdot)$ is a continuous function, then, by (S3) and applying the dominated convergence theorem, it follows that, for each fixed $t \geq 0$,

$$
\begin{aligned}
u_{1}(t) & =\lim _{n \rightarrow \infty} H_{f}^{n+1}(\widetilde{G})(t) \\
& =\lim _{n \rightarrow \infty} \int_{0}^{t} f\left(H_{f}^{n}(\widetilde{G})(t-s)\right) \mathrm{d} G(s) \\
& =\int_{0}^{t} f\left(\lim _{n \rightarrow \infty} H_{f}^{n}(\widetilde{G})(t-s)\right) \mathrm{d} G(s) \\
& =\int_{0}^{t} f\left(u_{1}(t-s)\right) \mathrm{d} G(s) \\
& =H_{f}\left(u_{1}\right)(t) .
\end{aligned}
$$

Moreover, since $v_{f}(\cdot)=H_{f}\left(v_{f}\right)(\cdot)$ and $u_{1}(\cdot)$ is bounded, $u_{1}(\cdot)=v_{f}(\cdot)$, because only one bounded function is a solution of (2.1) (see [3, p. 139]). The statement for the function $u_{2}(\cdot)$ follows similarly.

Proof of Theorem 2.2. Since $v_{f}(\cdot)$ is a distribution function and $f(s) \leq g(s)$ for all $0 \leq$ $s \leq 1$, then, for each $t \geq 0$,

$$
H_{f}\left(v_{f}\right)(t) \leq H_{g}\left(v_{f}\right)(t)
$$


Taking this fact into account and (2.1), we have $v_{f}(t) \leq H_{g}\left(v_{f}\right)(t)$ for all $t \geq 0$. Moreover, by (S2) in the proof of Theorem 2.1 and again taking iterations, for all $n \geq 1$ and $t \geq 0$, we obtain

$$
v_{f}(t) \leq H_{g}^{n}\left(v_{f}\right)(t),
$$

and the proof is completed using Theorem 2.1.

Remark 4.1. We note that the proofs of Theorem 2.1 and Theorem 2.2 hold even when $m_{f}>1$.

Proof of Theorem 2.3. We show by induction on $n$, for each $n \geq 1$, that, for all $t \geq 0$,

$$
\left|H_{f}^{n}(G)(t)-H_{g}^{n}(G)(t)\right| \leq \varepsilon\left(1-m_{f}^{n}\right)
$$

For fixed $t \geq 0$ and $\delta=\varepsilon\left(1-m_{f}\right)$, since $G(\cdot)$ is a DF for $n=1$, we deduce that

$$
\left|H_{f}(G)(t)-H_{g}(G)(t)\right| \leq \int_{0}^{t}|f(G(t-s))-g(G(t-s))| \mathrm{d} G(s) \leq \varepsilon\left(1-m_{f}\right) .
$$

By the induction hypothesis, (4.2) holds for $n$. Then, for $n+1$, we have

$$
\begin{aligned}
\left|H_{f}^{n+1}(G)(t)-H_{g}^{n+1}(G)(t)\right| \leq & \left|H_{f}\left(H_{f}^{n}(G)\right)(t)-H_{f}\left(H_{g}^{n}(G)\right)(t)\right| \\
& +\left|H_{f}\left(H_{g}^{n}(G)\right)(t)-H_{g}\left(H_{g}^{n}(G)\right)(t)\right| .
\end{aligned}
$$

By (S1) and (S2) in the proof of Theorem 2.1 and iterating, we deduce, for all $n \geq 1$, that $H_{f}^{n}(G)(t) \leq 1$ and $H_{g}^{n}(G)(t) \leq 1$. Taking these facts into account, we obtain

$$
\begin{aligned}
\left|H_{f}\left(H_{f}^{n}(G)\right)(t)-H_{f}\left(H_{g}^{n}(G)\right)(t)\right| & \leq \int_{0}^{t}\left|f\left(H_{f}^{n}(G)(t-s)\right)-f\left(H_{g}^{n}(G)(t-s)\right)\right| \mathrm{d} G(s) \\
& \leq m_{f} \sup _{0 \leq s^{*}<\infty}\left|H_{f}^{n}(G)\left(s^{*}\right)-H_{g}^{n}(G)\left(s^{*}\right)\right| \\
& \leq \varepsilon\left(1-m_{f}^{n}\right) m_{f}
\end{aligned}
$$

and

$$
\begin{aligned}
\left|H_{f}\left(H_{g}^{n}(G)\right)(t)-H_{g}\left(H_{g}^{n}(G)\right)(t)\right| & \leq \int_{0}^{t}\left|f\left(H_{g}^{n}(G)(t-s)\right)-g\left(H_{g}^{n}(G)(t-s)\right)\right| \mathrm{d} G(s) \\
& \leq \varepsilon\left(1-m_{f}\right) .
\end{aligned}
$$

Therefore, we conclude that

$$
\left|H_{f}^{n+1}(G)(t)-H_{g}^{n+1}(G)(t)\right| \leq \varepsilon\left(1-m_{f}^{n}\right) m_{f}+\varepsilon\left(1-m_{f}\right)=\varepsilon\left(1-m_{f}^{n+1}\right) .
$$

Since $m_{f}<1$, from (4.2), by applying Theorem 2.1, we obtain

$$
\sup _{0 \leq t<\infty}\left|v_{f}(t)-v_{g}(t)\right| \leq \varepsilon
$$

and this completes the proof.

Proof of Theorem 3.1. Let $p$ be such that $0<p<1$.

(i) Let $\alpha_{1}$ and $\alpha_{2}$ be such that $\alpha_{\text {inf }} \leq \alpha_{1}<\alpha_{2} \leq 1$. Taking into account the stochastic monotonicity property of the extinction time, we obtain

$$
p \leq v_{\alpha_{1}}\left(t_{p}^{\alpha_{1}}\right) \leq v_{\alpha_{2}}\left(t_{p}^{\alpha_{1}}\right)
$$

and, therefore, by the definition of $t_{p}^{\alpha_{2}}$, we deduce that $t_{p}^{\alpha_{2}} \leq t_{p}^{\alpha_{1}}$. 
(ii) Let $\alpha$ be such that $0<m_{\alpha}<m_{\alpha_{\text {inf }}}$. From the previous part, we guarantee the existence of $\lim _{\widetilde{\alpha} \rightarrow \alpha^{+}} t_{p}^{\widetilde{\alpha}}$, which is equal to $\bar{t}=\sup \left\{t_{p}^{\widetilde{\alpha}}: \widetilde{\alpha}>\alpha\right\}$. Therefore, $\bar{t} \leq t_{p}^{\alpha}$. On the other hand, from the continuity property of the extinction time, we deduce that, for each $\varepsilon>0$, there exists $\eta=\eta(\varepsilon, \alpha)>0$ such that

$$
p-\varepsilon \leq v_{\widetilde{\alpha}}\left(t_{p}^{\widetilde{\alpha}}\right)-\varepsilon \leq v_{\alpha}\left(t_{p}^{\widetilde{\alpha}}\right) \leq v_{\alpha}(\bar{t})
$$

for all $\widetilde{\alpha}$ such that $0<\widetilde{\alpha}-\alpha \leq \eta$. Then $p \leq v_{\alpha}(\bar{t})$, and so $t_{p}^{\alpha}=\bar{t}$.

(a) Applying the first part, we deduce that $\lim _{\widetilde{\alpha} \rightarrow \alpha^{-}} t_{p}^{\alpha}$ exists, that it is equal to $\underline{t}=$ $\inf \left\{t_{p}^{\widetilde{\alpha}}: \widetilde{\alpha}<\alpha\right\}$, and that $t_{p}^{\alpha} \leq \underline{t}$. Next, we prove that $\underline{t} \leq t^{*}$. We split the proof into two cases: $v_{\alpha}\left(t^{*}\right)>p$ and $v_{\alpha}\left(t^{*}\right)=p$. First we consider the case in which $v_{\alpha}\left(t^{*}\right)>p$. Let $\varepsilon=v_{\alpha}\left(t^{*}\right)-p$. From the continuity property of the extinction time we deduce that there exists $\eta=\eta(\varepsilon, \alpha)>0$ such that

$$
v_{\alpha}\left(t^{*}\right)-v_{\widetilde{\alpha}}\left(t^{*}\right) \leq \varepsilon=v_{\alpha}\left(t^{*}\right)-p
$$

for all $\widetilde{\alpha}, 0<\alpha-\widetilde{\alpha} \leq \eta$. Then $p \leq v_{\widetilde{\alpha}}\left(t^{*}\right)$ and, therefore, we have $t_{p}^{\widetilde{\alpha}} \leq t^{*}$ and, consequently, $\underline{t} \leq t^{*}$.

Finally, we consider the case in which $v_{\alpha}\left(t^{*}\right)=p$. By the definition of $t^{*}$ we have $p<v_{\alpha}(t)$ for all $t>t^{*}$. For each $t>t^{*}$, let $\varepsilon=v_{\alpha}(t)-p$. From the continuity property of the extinction time, we deduce that there exists $\eta=\eta(\varepsilon, \alpha)>0$ such that

$$
v_{\alpha}(t)-v_{\widetilde{\alpha}}(t) \leq \varepsilon=v_{\alpha}(t)-p
$$

for all $\widetilde{\alpha}, 0<\alpha-\widetilde{\alpha} \leq \eta$. Then $p \leq v_{\widetilde{\alpha}}(t), t_{p}^{\widetilde{\alpha}} \leq t$, and $\underline{t} \leq t$, and, consequently, $\underline{t} \leq t^{*}$.

(b) This part is proved similarly to the $v_{\alpha}\left(t^{*}\right)>p$ case above, replacing $t^{*}$ by $t_{p}^{\alpha}$.

(c) From (a) we obtain $\lim _{\widetilde{\alpha} \rightarrow \alpha^{-}} t_{p}^{\widetilde{\alpha}}=t_{p}^{\alpha}$, and this completes the proof.

\section{Appendix A}

We consider a BHBP initiated with one individual, with reproduction law $\left\{p_{k, \alpha}\right\}_{k \geq 0}$, where $0 \leq \alpha \leq 1$, DF $G^{*}(\cdot)$ of the initial progenitor's life length, and DF $G(\cdot)$ of the life length for other individuals. We suppose that $G^{*}(t) \geq G(t)$ for all $t \geq 0$. In an epidemiological context, this condition reflects the fact that the life-length distribution $G^{*}(\cdot)$ of the initial individual after vaccination is always less than or equal to its total life length, given by $G(\cdot)$.

We denote by $\widehat{T}_{\alpha}$ the extinction time of such a BHBP. Also, we denote by $\widehat{v}_{\alpha}(\cdot)$ the DF of the extinction time $\widehat{T}_{\alpha}$, i.e. $\widehat{v}_{\alpha}(t)=\mathrm{P}\left(\widehat{T}_{\alpha} \leq t\right)$ for all $t \in \mathbb{R}$. Following a heuristic derivation as in [3, p. 138], we obtain the integral equation

$$
\widehat{v}_{\alpha}(t)=\int_{0}^{t} f_{\alpha}\left(v_{\alpha}(t-s)\right) \mathrm{d} G^{*}(s), \quad t \geq 0 .
$$

From (4.1) and (A.1), for all $t \geq 0$, we obtain

$$
v_{\alpha}(t)=f_{\alpha}(0) G(t)+\left(1-f_{\alpha}(0)\right)\left(F_{\alpha} * G\right)(t)
$$

and

$$
\widehat{v}_{\alpha}(t)=f_{\alpha}(0) G^{*}(t)+\left(1-f_{\alpha}(0)\right)\left(F_{\alpha} * G^{*}\right)(t),
$$

where $F_{\alpha} * G^{*}(\cdot)$ denotes the convolution of $F_{\alpha}(\cdot)$ and $G^{*}(\cdot)$, with

$$
F_{\alpha}(y)=\left(1-f_{\alpha}(0)\right)^{-1}\left(f_{\alpha}\left(v_{\alpha}(y)\right)-f_{\alpha}(0)\right) \text { for all } y \geq 0 .
$$


Since $G^{*}(t) \geq G(t)$ for all $t \geq 0$, then

$$
\left(F_{\alpha} * G^{*}\right)(t) \geq\left(F_{\alpha} * G\right)(t)
$$

for all $t \geq 0$ and, therefore, $\widehat{v}_{\alpha}(t) \geq v_{\alpha}(t)$ for all $t \geq 0$.

\section{Acknowledgements}

The authors thank Dr M. Rodríguez-Arias for his advice about integral equations, which helped improve the results given in this paper, and the anonymous referee for her/his comments and suggestions.

Research partially supported by the Ministerio de Educación y Ciencia and the FEDER through the Plan Nacional de Investigación Científica, Desarrollo e Innovación Tecnológica, grants MTM2006-08891 and MTM2009-13248, by the NFSI, grant VU-MI-105/2005, Bulgaria, and by an action of the program ECO-NET'2006 financed by the French government.

\section{References}

[1] Agresti, A. (1974). Bounds on the extinction time distribution of a branching process. Adv. Appl. Prob. 6, 322-335.

[2] Andersson, H. And Britton, T. (2000). Stochastic Epidemic Models and Their Statistical Analysis (Lecture Notes Statist. 151). Springer, New York.

[3] Athreya, K. B. ANd Ney, P. E. (1972). Branching Processes. Springer, Berlin.

[4] Axelrod, D. E. And Gusev, Y. and Gamel, J. W. (1997). Ras oncogene-transformed and nontransformed cell population are each heterogeneous but respond differently to the chemotherpeutic drug cytosine arabinoside (Ara-C). Cancer Chem. Pharm. 39, 445-451.

[5] Axelrod, D. E. And Gusev, Y. And KuczeK, T. (1993). Persistence of cell cycle times over many generations as determined by heritability of colony sizes of ras oncogene-transformed and non-transformed cells. Cell Prolif. 26, 235-249.

[6] Ball, F. And Donnelley, P. (1995). Strong approximations for epidemic models. Stoch. Process. Appl. 55, 1-21.

[7] Barbour, A. D. (1975). The duration of the closed stochastic epidemic. Biometrika 62, 477-482.

[8] Becker, N. G. And Britton, T. (2004). Estimating vaccine efficacy from small outbreaks. Biometrika 91, 363-382.

[9] Billingsley, P. (1986). Probility and Measure, 2nd edn. John Wiley, New York.

[10] Farrington, C. P. and Grant, A. D. (1999). The distribution of time to extinction in subcritical branching processes: applications to outbreaks of infectious disease, J. Appl. Prob. 36, 771-779.

[11] Farrington, C. P. and Kanaan, M. N. and Gay, N. J. (2003). Branching process models for surveillance of infectious diseases controlled by mass vaccination. Biostatist. 4, 279-295.

[12] Gusev, Y. and Axelrod, D. E. (1995). Evaluation of models of inheritance of cell cycle times: computer simulation and recloning experiments. In Mathematical Population Dynamics: Analysis of Heterogeneity, Vol. 2, Wuerz Publications, Winnipeg, pp. 97-116.

[13] Haccou, P. And Jagers, P. And Vatutin, V. A. (2007) Branching Processes: Variation, Growth, and Extinction of Populations. Cambridge University Press.

[14] Heinzmann, D. (2009). Extinction times in multitype Markov branching processes. J. Appl. Prob. 46, $296-307$.

[15] Isham, V. (2005). Stochastic models for epidemics. In Celebrating Statistics (Oxford Statist. Sci. Ser. 33), Oxford University Press, pp. 27-54.

[16] Johnson, R. A. And Susarla, V. and Van Ryzin, J. (1979). Bayesian nonparametric estimation for agedependent branching processes. Stoch. Process. Appl. 9, 307-318.

[17] Kimmel, M. (1985). Nonparametric analysis of stathmokinesis. Math. Biosci. 74, 111-123.

[18] Kimmel, M. and Axelrod, D. E. (2002). Branching Processes in Biology. Springer, New York.

[19] Kimmel, M. and Traganos, F. (1986). Estimation and prediction of cell cycle specific effects of anticancer drugs. Math. Biosci. 80, 187-208.

[20] Martínez, R. and Slavtchova-Bojkova, M. (2005). Comparison between numerical and simulation methods for age-dependent branching models with immigration. Pliska Stud. Math. Bulgar. 17, 147-154.

[21] Mode, C. J. and Sleemam, C. K. (2000). Stochastic Processes in Epidemiology. World Scientific, Singapore.

[22] NÅsell, I. (2002). Stochastic models of some endemic infections. Math. Biosci. 179, 1-19. 
[23] Pakes, A. G. (1989). On the asymptotic behaviour of the extinction time of the simple branching process. Adv. Appl. Prob. 21, 470-472.

[24] Pakes, A. G. (2003). Biological applications of branching processes. In Stochastic Processes: Modelling and Simulation (Handbook Statist. 21), eds D. N. Shanbhag and C. R. Rao, North Holland, Amsterdam, pp. 693-773.

[25] R Development Core Team (2009). R: A Language and Environment for Statistical Computing. R Foundation for Statistical Computing, Vienna, Austria.

[26] Yakovlev, A. And Yanev, N. (2006). Branching Stochastic Processes with immigration in analysis of renewing cell populations. Math. Biosci. 203, 37-63.

[27] Yakovlev, A. And Yanev, N. (2007). Age and residual lifetime distributions for branching processes. Statist. Prob. Lett. 77, 503-513. 